\title{
Publisher correction: The spatial scales of species coexistence
}

Simon P. Hart, Jacob Usinowicz and Jonathan M. Levine

Nature Ecology \& Evolution 1, 1066-1073 (2017); published online 21 July 2017; corrected online 2 August 2017

An error during production led to a truncation of the final two sentences in the abstract, which should have read 'In so doing, this framework substantially reframes current approaches to spatial community ecology. Quantifying the spatial scales of species coexistence will permit the next important advance in our understanding of the maintenance of diversity in nature, and should improve the contribution of community ecology to biodiversity conservation.' These have been corrected in all versions of the Perspective. 